\title{
Triple procedure; analysis of outcome, refraction, and intraocular lens power calculation
}

Annette J M Geerards, Elissa Hassmann, W Houdijn Beekhuis, L Remeyer, Gabriel van Rij, Wilhelmina J Rijneveld

\begin{abstract}
Aims-A total of 97 triple procedures performed over a 6 year period were studied retrospectively to determine the best approach to calculate intraocular lens power.

Methods-The cases were divided into two diagnostic categories.

Results-After 1 year best corrected visual acuity was $20 / 40$ or better in $37.5 \%$ of the cases of the 'modified group'. This group consists of patients with the diagnosis Fuchs' dystrophy, non-guttate endothelial dystrophy, and Reis-Buckler dystrophy. Analysis of visual acuity was made using $\log$ MAR. A final postoperative refraction within 2 dioptres of predicted refraction was achieved in $76.5 \%$ of patients in the modified group.

Conclusion-In future, in the absence of a keratometry, a keratometry value of 7.49 mm will be used for calculation of the power of the implant as analysed in this study.

(Br F Ophthalmol 1997;81:774-777)
\end{abstract}

When a patient requires both penetrating keratoplasty and cataract extraction the two procedures can be performed in combination or separately, with or without an intraocular lens implant. Combined cataract extraction and penetrating keratoplasty has been reported by Katzin and Meltzer in 1966. ${ }^{1}$ In 1976, intraocular lens implantation was added to this procedure. ${ }^{2-4}$ Initially, iris fixated lenses were used. Later, the introduction of posterior chamber lenses as well as refined extracapsular surgical techniques ${ }^{2}$ improved the results of the triple procedure. ${ }^{5}$ Compared with a staged procedure, usually at a 6-12 month interval, the combined or triple procedure allows faster visual rehabilitation without compromising graft survival. ${ }^{167}$

Despite the success of the triple procedure, there is still no precise formula available to best predict the intraocular lens power needed to produce postoperative emmetropia. Katz and Forster have demonstrated that neither keratometry readings from the operated eye nor the ones from the fellow eye provide predictable refractive results after a triple procedure. $^{8}$

The purpose of this study was to evaluate our data using our modified formula to determine whether or not a more accurate formula could be derived to calculate intraocular lens power for postoperative emmetropia with the triple procedure.

\section{Materials and methods}

In this study we retrospectively reviewed the results of 109 eyes of 97 patients who underwent an elective triple procedure between 1985 and 1991. These patients were randomly sampled from our cornea clinic. Ninety seven eyes were used for statistical analysis. Twelve eyes were eliminated to avoid patient bias as they were second eyes of patients in the study. The surgeries were performed by one of three surgeons (GvR, HB, AR). The patients, 35 men and 74 women, ranged in age from 43 to 91 years (mean 72.5 years). The preoperative diagnosis, listed in Table 1, included cataract and endothelial dystrophy in $45 \%$ of cases, and cataract and interstitial keratitis in $20 \%$ of cases. Herpes simplex scars and a mixture of other diagnoses made up the remaining $35 \%$.

Owing to the differing diagnoses, a modified group was selected including the patients with Fuchs' dystrophy, non-guttate endothelial dystrophy, and Reis-Buckler dystrophy. Fifty one patients were in this group and separate calculations on the postoperative results were done on these patients.

Preoperative axial lengths were measured using a Sonomed ultrasound (A-2000, Sonomed technology). Keratometry values were obtained from both eyes when possible.

In 39 cases, keratometry values were not obtainable because of an irregular corneal surface. If keratometry values of the operated eye were not available, those of the fellow eye were used. When no keratometry values were available, the standard value of $7.0 \mathrm{~mm}$ (48 D) was used. Intraocular lens power was calculated for emmetropia using the Colenbrander formula. ${ }^{9}{ }^{10}$ In some cases the power was calculated to achieve a mild myopic or hyperopic

Table 1 Preoperative diagnosis

\begin{tabular}{lc}
\hline Diagnosis & Number (\%) \\
\hline Fuchs' dystrophy & $44(45.4)$ \\
Interstitial keratitis & $20(20.6)$ \\
Herpes simplex keratitis & $10(10.3)$ \\
Keratoconus & $1(1.0)$ \\
Trauma & $4(4.1)$ \\
Macula corneas & $3(3.1)$ \\
Bullous keratopathy & $3(3.1)$ \\
Ulcus corneas & $1(1.0)$ \\
Herpes zoster & $1(1.0)$ \\
Non-guttate endothelial dystrophy & $6(6.2)$ \\
Reis-Buckler dystrophy & $1(1.0)$ \\
Unknown & $4(4.1)$ \\
\hline
\end{tabular}


Table 2 Donor-recipient disparity versus refractive values (SD) in all patients

\begin{tabular}{llll}
\hline Donor-recipient & $\begin{array}{l}7-7.5 \mathrm{~mm} \\
n=31\end{array}$ & $\begin{array}{l}7.5-8.0 \mathrm{~mm} \\
n=51\end{array}$ & $\begin{array}{l}8-8.5 \mathrm{~mm} \\
n=8\end{array}$ \\
\hline Lens power & $20.33(3.96)$ & $18.52(3.29)$ & $19.67(5.51)$ \\
Predicted refraction & $+1.05(1.10)$ & $+1.10(1.73)$ & $+2.39(1.12)$ \\
SE 1 & $-0.88(2.81)$ & $-1.08(1.97)$ & $+1.08(0.52)$ \\
SE 2 & $-0.56(2.98)$ & $-1.94(3.00)$ & $+0.08(2.38)$ \\
SE 3 & $-1.30(4.42)$ & $-0.70(2.16)$ & $-2.75^{\star}$ \\
SE 4 & $6.25^{\star}$ & $0.50(4.23)$ & $-1.38(0.88)$ \\
\hline
\end{tabular}

$\mathrm{SE}=$ spherical equivalent. ${ }^{\star}$ Only one observation.

refraction to avoid significant anisometropia. Changes in depth of anterior chamber or axial length were not taken into account.

The surgical technique used by the three surgeons was similar. The pupil was dilated preoperatively with tropicamide $1 \%$ and phenylephrine $5 \%$. All patients were operated under general anaesthesia. The donor corneas were preserved in MEM medium. ${ }^{11}$

A Flieringa ring secured by six scleral sutures was used for scleral support and eyelid retraction. The recipient corneal bed was prepared and ranged from $6.1 \mathrm{~mm}$ to $8 \mathrm{~mm}$. The donor cornea was cut endothelial side up using a disposable guillotine trephine (Medical Workshop, Groningen, Netherlands). The donor was oversized by $0.5 \mathrm{~mm}$ in all cases. The recipient cornea was then partially incised using a disposable trephine (Hessburg Barron, Katena Products, Denville, NJ, USA). The anterior chamber was then entered using a disposable razor blade (Alcon, Fort Worth, TX, USA). Curved corneal scissors were used to completely excise the corneal button.

Anterior capsulorrhexis was performed with a pre-bent needle. Balanced saline solution was used for hydrodissection of the nucleus. Two horizontal cuts were made at 3 and 9 o'clock positions of the anterior capsule with fine scissors. The nucleus was delivered by gentle pressure at the limbus, engaging the nucleus with the blade. The lens cortex was removed by manual irrigation and aspiration.

The desired power of the posterior chamber intraocular lens was selected and, after rinsing with balanced salt solution, the lens was implanted. Occasionally, viscoelastics were used when there was a narrow anterior chamber to separate iris from cornea during suturing.

The donor cornea was sutured into the recipient bed with eight interrupted 10/0 nylon sutures, followed by a 24 bite continuous $11-0$ nylon suture. In cases with a vascularised recipient bed, 16 interrupted 10-0 nylon sutures were used. The wound was checked for water tightness. The suture knots were trimmed and buried into the host cornea.

Dexamethasone and gentamicin were injected subconjunctivally in all cases.

All patients were placed on a postoperative tapering regimen of dexamethasone $0.1 \%$ and chloramphenicol $0.4 \%$ The interrupted sutures were removed selectively to reduce astigmatism. ${ }^{12} 13$

All patients were followed for a minimum of 1 year. Postoperative keratometry values and corneal radius were measured at each visit. Best corrected visual acuity measured by an ophthalmologist was also obtained at each visit and expressed as Snellen visual acuity. Spherical equivalent values were used in all calculations. Mean visual acuity was calculated using the logarithm of the minimum angle of resolution $^{14}{ }^{15}(\log M A R)$. The predicted postoperative refraction was compared to the actual stable postoperative refraction. These values were compared with the calculated lens power and to the ideal lens power that would have achieved emmetropia more closely.

All postoperative observations were divided into four periods. We assembled data for period 1 (first 3 months after operation), period 2 (4-12 months), period 3 (13 to 24 months), and period 4 ( 25 months or more).

Statistical analysis was performed by a statistician using sPSS. Statistical significance was calculated using the Mann-Whitney, Student's $t$ test or Kruskal-Wallis test.

\section{Results}

The median best corrected preoperative visual acuity was less than $20 / 100$ in the studied population of 97 patients. The average preoperative spherical equivalent was 0.43 (SD 3.6) dioptres, and the average preoperative keratometric cylinder was $0.88 \mathrm{D}$.

The best corrected visual acuity after 1 year was $20 / 40$ or better in $37.5 \%$ of cases, $20 / 40$ to $20 / 60$ in $40 \%$ of cases, and $<20 / 60$ in $22.5 \%$ of cases. Of the nine patients with visual acuity $<20 / 60,3 / 9(30 \%)$ had pre-existing macular pathology. Four out of nine $(44 \%)$ had corneal opacities from recurrent herpetic keratitis.

Postoperative complications included phthisis after traumatic penetration to the graft (one case), herpes simplex on a graft in a patient with no previous history of herpes simplex virus (one case), bacterial keratitis in the graft (four cases), and one irreversible allograft reaction. Twenty seven patients required a post operative YAG laser posterior capsulotomy. Because of too high astigmatism in the graft, five patients underwent a surgical astigmatism correction. Subsequent glaucoma filtering surgery was required in one Fuchs' patient due to secondary glaucoma.

In Table 2, the spherical equivalents of the postoperative refractive values of the whole group are given. Comparison is made between the donor-recipient disparity and the predicted refraction, and the postoperative refraction. No statistically significant difference was found between the various donor/recipient sizes in lens power and refraction nor was there any difference at the intervals.

At 1 year follow up 33/51 (65\%) of the patients in the modified group improved in visual acuity. After 2 years, 13/19 (68\%) had an improved postoperative visual acuity. Both results are statistically significant ( $\mathrm{p}$ value $<0.005$ ). Table 3 presents the postoperative refractive results and their spherical equivalents of the modified group. No statistical significance was found between our modified group and the entire sample with respect to these values. In addition, no statistical significance was detected within the modified group when comparing the spherical equivalents of 
Table 3 Postoperative refractive values (SD) of the modified group

\begin{tabular}{lllll}
\hline & 0-3 months & 3-12 months & 12-24 months & $>24$ months \\
\hline Visual acuity & $0.25(0.15)$ & $0.51(0.25)$ & $0.52(0.24)$ & $0.45(0.24)$ \\
Pinhole & $0.33(0.19)$ & $0.43(0.20)$ & $0.52(0.19)$ & $0.63(0.21)$ \\
Cylinder (D) & $4.90(2.40)$ & $3.07(2.13)$ & $2.90(2.80)$ & $2.27(1.98)$ \\
Sphere (D) & $+1.26(2.53)$ & $+0.28(2.90)$ & $+0.01(2.05)$ & $+1.70(4.68)$ \\
k Value & & & $7.49(0.44)$ & \\
$\begin{array}{l}\text { Mean keratometic } \\
\quad \text { cylinders (D) }\end{array}$ & $5.44(2.90)$ & $3.5(2.12)$ & $4.16(3.39)$ & $2.77(1.97)$ \\
SE (D) & $-0.84(2.26)$ & $-1.10(3.05)$ & $-0.98(2.71)$ & $+0.68(4.07)$ \\
\hline
\end{tabular}

${ }^{\star}$ Snellen acuity.

Table 4 Data of the modified group after 1 year (SD) in dioptres

\begin{tabular}{ll}
\hline Lens power used & $18.86(3.71)$ \\
Predicted spherical equivalent & $+1.68(1.58)$ \\
Calculated spherical equivalent $\dagger$ & \\
$\quad(\mathrm{K}=7.49)$ & $+0.57(2.06)$ \\
Resultant spherical equivalent $\ddagger$ & $-0.98(2.71)$ \\
Range of errors & -6.00 to +4.50 \\
Within +/- 2 D of emmetropia (\%) & $39 / 51(76.5)$
\end{tabular}

*Spherical equivalent calculated according to the computer outcome.

† Spherical equivalent calculated using the mean keratometry value of $7.40 \mathrm{~mm}$

$\ddagger$ Spherical equivalent calculated according to the refraction after 1 year.

the selected postoperative periods. The average keratometry value after 1 year of the modified group did not statistically differ from the entire group of patients being $7.49 \mathrm{~mm}$ (45 D, SD $0.44 \mathrm{~mm})$.

Intraocular lens power varied from 6 to 30 dioptres. In our modified group, mean power of implanted intraocular lens was 18.86 dioptres (SD 3.71). Predicted spherical equivalent was +1.68 (SD 1.58) and $\mathrm{k}$ value between 1 and 2 years postoperative was $7.49 \mathrm{~mm}$ (45 dioptres, SD $0.44 \mathrm{~mm}$ ) (Table 4 ). The average axial length was $24.16 \mathrm{~mm}$ for the entire group and $23.73 \mathrm{~mm}$ in the modified group. This difference is not statistically significant. Axial lengths in interstitial keratitis patients differed statistically from those of the Fuchs' patients and herpetic patients.

No statistical difference could be proved between the different diagnoses.

\section{Discussion}

In order to calculate the power of an intraocular lens in a triple procedure for a certain refraction, either myopia or iseikonia, many relations between pre- and postoperative variables need to be evaluated. In determining the power of the intraocular lens, axial length is the most important factor. ${ }^{16}$ Most ultrasonic axial length measurements are accurate within 0.2 $\mathrm{mm}(0.6 \mathrm{D})$. The remaining variables in determining intraocular lens power are postoperative keratometry and anterior chamber depth. ${ }^{17}$ In this study, we attempted to evaluate our data to help develop a more accurate formula for calculating intraocular lens power when planning a triple procedure. Our current method of estimating intraocular lens power uses the Binkhorst formula, thereby assuming $7.0 \mathrm{~mm}$ for the postkeratoplasty radius to achieve emmetropia in case it is impossible to use preoperative values. The refractive results following a combined penetrating keratoplasty and cataract extraction with intraocular lens implantation in our institution were divided into two groups.

Patients with a diagnosis of Fuchs's dystrophy, non-guttate endothelial dystrophy, and Reis-Buckler formed the modified group. This division was made since in these patients usually both eyes are affected, which makes it possible to use $\mathrm{k}$ readings of the other eye if necessary. Therefore, in this group postoperative calculations are made to induce slight myopia versus emmetropia for monocular diagnoses. For patients in this group the prognosis is fairly good not only on a clear graft but also with respect to visual acuity and astigmatism.

Of our modified group, $76.5 \%$ had a postoperative refraction within $2 \mathrm{D}$ of emmetropia. This compares favourably with the results of a study by Binder ${ }^{16}$ in which he compared his results on 77 triple procedures with other authors. In his study, 38 of 68 (55.8\%) eyes were within $2 \mathrm{D}$ of emmetropia. The range of refractive error in the modified group in our study was $-6.00 \mathrm{D}$ to $+4.50 \mathrm{D}$, which is smaller than in other studies. We feel this is an important factor in visual rehabilitation. Some surgeons are in favour of a two staged procedure $^{19}$ to optimise the final refraction. Distribution of spherical equivalent refractive errors in the latter study was better than our results but there were more intraoperative complications.

Mean visual acuity was calculated using the logarithm of the minimum angle of resolution. ${ }^{14}{ }^{15}$ Visual acuity after triple procedure improved in $68 \%$ of our patients in the modified group. Twenty five per cent demonstrated no improvement. The best corrected visual acuity was more than $20 / 40$ in $37.5 \%$ of the cases, which is worse than reported in other studies. ${ }^{16}{ }^{19-22}$ This does not compare favourably to the results by several surgeons who report a clear graft and visual acuity $>20 / 40$ in $68-77 \%$ of cases. $^{1619-21}$ The mean Snellen visual acuity after 1 year was higher for all periods tested compared with the acuity with the MAR. As is shown by Westheimer ${ }^{14}$ and Holladay and Prager, ${ }^{15}$ visual performances of both high and low visual acuities are predicted equally well using the log MAR. Therefore, our conclusions based on potential visual acuity are relatively correct.

Spherical equivalent after 1 year was -0.98 $\mathrm{D}$ in our modified population. Calculations done retrospectively with the power of the intraocular implant and the mean keratometry value of this group after one year gives a slight hyperopia-that is, +0.57 D. The predicted spherical equivalent was also slightly hyperopic $(+1.68 \mathrm{D})$ (Table 4). Although this is not statistically significant, we like to discuss a few variables causing deviations from the refraction calculated. Firstly, although calculations are done with preoperative and postoperative values for anterior chamber depth, a slightly forward or backward placed lens or instability of the zonules will change final refraction. Placing the lens within the capsular bag or within the sulcus can decrease or increase the effective power of the lens from 0.5 to $1.0 \mathrm{D}^{23}$ 
Axial length measurements are within 0.2 $\mathrm{mm}$ which results in differences in effective refraction of more than $0.5 \mathrm{D}$. Graft-host disparity did not affect the final refraction significantly but a considerable standard deviation in the final sferical equivalent was seen (Table 3). All these factors contribute to the range of errors.

Postoperative corneal astigmatism can limit the visual outcome significantly after penetrating keratoplasty. Factors affecting astigmatism which cannot be foreseen, are individual wound healing, and reaction on sutures, like cheesewiring. Furthermore, oval trephination of the recipient may be an unpredictable factor in postkeratoplasty astigmatism. ${ }^{24}$ In our study, astigmatism after 1 year was $4.16 \mathrm{D}$, with an individual excess of $12 \mathrm{D}$. Refractive cylinder was $2.90 \mathrm{D}$, which is not statistically different from keratometric cylinder. These data can be compared to the large study done by Stanford et $a .^{25}$ One of the well known factors influencing postkeratoplasty astigmatism is different suturing by different surgeons. Although our three corneal surgeons used the same technique, we were curious to know about their individual keratometry after 1 year. Our results concerning postoperative keratometry for the different surgeons were within 1 dioptre which is not statistically significant nor was the astigmatism between the three surgeons statistically significant. Suture removal can change not only astigmatism to a large extent but also refraction with a tendency to myopia. Patients operated in our clinic were usually sent to their referring ophthalomologist after a year without taking out the sutures. Those who remained under our control were only taken out when complications as suture infiltrate occurred and otherwise at least 2 years after operation. Follow up data of these patients are inconsistent.

We could not find any significant relation in the different donor-recipient disparity either, although we make no calculations for keratometry, but for spherical equivalent. In our opinion, this gives earlier significance as we hypothesised that larger grafts relatively give a flatter cornea and smaller total axial length which both account for a less myopic tendency. This could explain the hyperopic tendency in our data although they are not significant, because of the small numbers in this group after 2 years $(n=3)$. Interestingly, Abdel-Hakim found both larger and smaller axial lengths in his study ${ }^{26}$ compared with the preoperative value. Unfortunately, he omitted measuring postoperative after the seventh case.

In conclusion, analysis of our results of the combined penetrating keratoplasty and cataract extraction with intraocular implant demonstrated a different postoperative keratometry reading than the one we used before. Nevertheless, results of postoperative spherical equivalent and percentage between +2 and -2
D of emmetropia were nevertheless satisfactory. In the future, calculations for intraocular lens power will be made using our specific calculated $\mathrm{K}$ reading $(7.49 \mathrm{~mm})$. We recommend that every surgeon constantly evaluates the data of his triple procedures and adjusts the lens power formula accordingly.

We are grateful to Brigitte Staal, MD, for collecting the data, and to Paul Mulder, PhD, Erasmus University of Rotterdam, for his statistical assistance.

1 Katzin HM, Meltzer JF. Combined surgery for corneal transplantation and cataract extraction. Am $\mathcal{f}$ Ophthalmol 1966;62:560.

2 Taylor DM. Keratoplasty and intraocular lenses. Ophthalmic Surg 1976;7:31-42.

3 Kramer SG. Penetrating keratoplasty combined with extracapsular cataract extraction. Am $\mathcal{F}$ Ophthalmol 1985; 100:129-33.

4 Groden LR. Continuous tear capsulotomy and phacoemulsification cataract extraction combined with penetrating sification cataract extraction combined with p
keratoplasty. Refract Corneal Surg 1990;6:458-9.

5 Brady SE, Rapuano CJ, Arentsen JJ, Cohen EJ, Lsibson PR. Clinical indications for and procedures associated with penetrating keratoplasty, 1983-1988. Am f Ophthalmol 1989;108:118-22.

6 Taylor DM, Khaligh A, Maxwell R. Keratoplasty and intraocular lenses: follow-up study. Ophthalmic Surg 1977; 8:49-57.

7 Buxton JN, Jaffe MS. Combined keratoplasty, cataract extraction and intraocular lens implantation. Am Intraocul Implant Soc J 1978;4:110

$8 \mathrm{Katz}$ HR, Forster RK. Intraocular lens calculation in combined penetrating keratoplasty, cataract extraction and intraocular lens implantation. Ophthalmology 1985;92: 1203-7.

9 Oguchi Y, Balen van ATM. Ultrasonic study of the refraction of patients with pseudophakos. Ultrasound Med refraction of patients

10 Heijde van der GL. The optical correction of unilateral aphakia. Trans Am Acad Ophthalmol Otol 1976;81:OP80-8.

11 Pels L, Suchard Y. Organ culture in the Netherlands; preservation and endothelial evaluation. In: Corneal surgery, theory, technique, and tissue, St Louis: Mosby, 1993:622-32.

12 Harris DJ, Waring GO, Burk LL. Keratography as a guide to selective suture removal for the reduction of astigmatism after penetrating keratoplasty. Ophthalmology 1989;96: 1597-607.

13 Musch DC, Meyer RF, Sugar A, Soong HK. Corneal astigmatism after penetrating keratoplasty. The role of suture technique. Ophthalmology 1989;96:698-703.

14 Westheimer G. Scaling of visual acuity measurements. Arch Ophthalmol 1979;97:327-30.

15 Holladay JT, Prager TC. Mean visual acuity. Am f Ophthalmol 1991;111:372-4.

16 Binder PS. Intraocular lens powers used in the triple procedure. Effect on visual acuity and refractive error. Ophthalmology 1985;92:1561-6.

17 Brightbill FS. Combined procedures. In: Corneal surgery, theory, technique, and tissue. St Louis: Mosby 1993:177-83.

18 Binder PS. The triple procedure. Refractive results. 1985 update. Ophthalmology 1986;93:1482-8.

19 Geggel HS. Intraocular lens implantation after penetrating keratoplasty. Improved unaided visual acuity, astigmatism, and safety in patients with combined corneal disease and cataract. Ophthalmology 1990;97:1460-7.

20 Crawford GJ, Doyle Stulting R, Waring GO, Van Meter WS, Wilson LA. The triple procedure. Analysis of outcome, refraction, and intraocular lens power calculation. Ophthalmology 1986;93:817-24.

21 Mattax JB, McCulley JP. The effect of standardized keratoplasty technique on IOL power calculation for the triple procedure. Acta Ophthalmol 1989;67:24-8.

22 Price FW, Whitson WE, Marks RG. Progression of visual acuity after penetrating keratoplasty. Ophthalmology 1991; 98:1177-85.

23 Binder PS. Refractive errors encountered with the triple procedure. In: Cornea, refractive surgery, and contact lens. Trans N Orleans Acad Ophthalmol 1987:111-20.

24 Perlman EM. An analysis and interpretation of refractive errors after penetrating keratoplasty. Ophthalmology 1981; $\mathbf{8 8 : 3 9 - 4 5 . ~}$

25 Stanford KD, Klesges LM, Wood TO. Simultaneous penetrating keratoplasty, extracapsular cataract extraction, and intraocular lens implantation. $\mathcal{F}$ Cataract Refract Surg 1991;17:824-9.

26 Abdel-Hakim ASE, Khalil A. Intraocular lens power calculations in the triple procedure. Br f Ophthalmol 1989;72: 709-13. 